\title{
Wave Optical Calculation of Probe Size in Low Energy Scanning Electron Microscope
}

\author{
Tomáš Radlička
}

Institute Scientific Instruments of the ASCR, v.v.i., Královopolská 147, 61264 Brno, Czech Republic

\begin{abstract}
A wave optical calculation of the probe size of a low energy scanning electron microscope is presented. The resolution for the optimal aperture was computed and compared with results of standard approaches. The effect of deflection aberrations is also considered, and it was found to be critical for the landing energies below $5 \mathrm{eV}$ and fields of view larger than $100 \times 100 \mu^{2}$.
\end{abstract}

\section{Introduction}

The resolution of the scanning electron microscope (SEM) depends on the probe size and on the interaction volume of the electrons within a sample. As the interaction volume depends on the sample type, it was not considered it in the study. Hence the probe size expresses the best possible resolution a system can have. The interaction volume decreases with decreasing landing energy, and for landing energies less than $10 \mathrm{eV}$ the probe size corresponds to the resolution very accurately.

There are several ways of determining the probe size. A very popular approach is the work by Barth $\&$ Kruit [1], which allows computing the probe size containing a given fraction of current (FC) of a round beam. Even if this approach is in a good agreement with wave optical calculations and experiments, it is not optimized for the use at very low energies of several $\mathrm{eV}$, for which the effect of the chromatic aberration is more critical.

Thus a complete wave optical calculation covering the second order chromatic aberration and the chromaticity of the spherical aberration seems necessary to determine the spot size correctly for low energies. The calculation also needs to include the effect of the deflection aberrations that become critical for low energies, mainly the effect of the dispersion increases.

\section{Wave optical calculation of the probe size}

We used wave optical calculations covering the effect of the axial aberrations of an electron beam of a finite brightness. As the resolution is mainly influenced by chromatic aberrations, we consider them up to the second degree, but only the effect of the primary geometrical aberrations is needed. We set from the current density generated by a point source in the object plane $\boldsymbol{q}_{o}=\left(x_{o}, y_{o}\right)$ - the point spread function (PSF), $j_{P S F} \propto|\psi|^{2}$. In the vicinity of the paraxial image $\boldsymbol{q}_{i p}=\left(x_{i p}, y_{i p}\right)$ of the point $\boldsymbol{q}_{o}$ the wave function $\psi$ can be written in the form [2,3]:

$$
\psi\left(\boldsymbol{q}_{i p}+\boldsymbol{d}\right)=C \iint_{A n g .} \exp \left(-\frac{i}{\hbar} g_{i} \chi\left(\boldsymbol{q}_{\boldsymbol{o}}, \boldsymbol{q}_{\boldsymbol{i} \boldsymbol{p}}^{\prime}\right)\right) e^{\frac{i}{\hbar} g_{i} \boldsymbol{q}_{i p}^{\prime} \boldsymbol{d}} \mathrm{d} \boldsymbol{q}_{i p}^{\prime},
$$

where $g_{i}$ is the kinetic momentum in the image, $\boldsymbol{q}_{i p}^{\prime}=\left(x_{i p}^{\prime}, y_{i p}^{\prime}\right)$ is the paraxial slope in the image and $\boldsymbol{d}$ is the deviation of the point where the PSF is evaluated from the paraxial image $\boldsymbol{q}_{i p}$ in the image plane. The aberration function $\chi$ has a direct relationship with the aberration polynomial in the parameterization by the position in the object and the paraxial slope in the image 


$$
\Delta \boldsymbol{q}_{i}\left(\boldsymbol{q}_{o}, \boldsymbol{q}_{i p}\right)=\frac{\partial \chi\left(\boldsymbol{q}_{o}, \boldsymbol{q}_{i p}^{\prime}\right)}{\partial \boldsymbol{q}_{i p}^{\prime}}
$$

Using the standard complex notation $w=x+i y$ and $\omega=x_{i p}^{\prime}+i y_{i p}^{\prime}$, the aberration function for the axial point in the image reads [3]:

$$
\chi=\mathcal{R}\left\{A_{o} \bar{\omega}+\frac{1}{2} C_{1} \omega \bar{\omega}+\frac{1}{2} A_{1} \bar{\omega}^{2}+B_{2} \omega^{2} \bar{\omega}+\frac{1}{3} A_{2} \bar{\omega}^{3}+\frac{1}{4} C_{3}(\omega \bar{\omega})^{2}+\frac{1}{4} A_{3} \bar{\omega}^{4}+S_{3} \omega^{3} \bar{\omega}\right\},
$$

where the notation by Haider for the axial aberration coefficients is followed.

The real electron source has an energy spread, which affects the system properties with chromatic aberrations. The formula for the aberration function must be extended to cover the chromatic aberration effect. It can be simply done by replacing the deflection coefficient $A_{0}$ by $A_{0}-C_{d} \delta$, defocus coefficient $C_{1}$ by $C_{1}-C_{C} \delta-C_{C 2} \delta^{2}$ and the spherical aberration coefficient $C_{3}$ by $C_{3}-C_{3 C} \delta$. $\delta$ denotes relative energy width in the image $\delta=\Delta E / E$ and the standard notation for the dispersion $\left(C_{d}\right)$, the chromatic aberration of the first $\left(C_{C}\right)$ and second order $\left(C_{C 2}\right)$ and for the chromaticity of the spherical aberration $\left(C_{3 C}\right)$ was used. We can assume that the electrons emitted with different energies are incoherent. The resulting PSF for the point source with the energy density function $\rho(E)$ takes the form

$$
j_{P S F}(\boldsymbol{q})=\int_{E} \rho(E) j_{P S F}(E ; \boldsymbol{q}) \mathrm{d} E
$$

The real source has also a nonzero diameter, which increases the final probe size. In that case the current density in the image can be expressed by the integration through the distribution in the source $\rho\left(\boldsymbol{q}_{o}\right)$

$$
j(\boldsymbol{q})=\int_{\text {source }} j_{P S F}\left(\boldsymbol{q}_{o} ; \boldsymbol{q}\right) \rho\left(\boldsymbol{q}_{o}\right) d^{2} \boldsymbol{q}_{o}
$$

As the size of the source is very small, the effect of the off-axial aberrations can be neglected and the previous formula is reduced to a simple 2D convolution of the PSF with the paraxial image of the distribution in the object $\rho_{p}(\boldsymbol{q})=\rho(\boldsymbol{q} / M)$ :

$$
j(\boldsymbol{q})=\int_{\text {source }} j_{P S F}(\widetilde{\boldsymbol{q}}) \rho_{p}(\boldsymbol{q}-\widetilde{\boldsymbol{q}}) d^{2} \widetilde{\boldsymbol{q}}=j_{P S F} * \rho_{p}
$$

For the axially symmetrical system the aberration function is reduced to:

$$
\chi_{a}(|\omega|, \delta)=\mathcal{R}\left\{\frac{1}{2}\left(C_{1}-C_{C} \delta-C_{C 2} \delta^{2}\right) \omega \bar{\omega}+\frac{1}{4}\left(C_{3}-C_{3 C} \delta\right)(\omega \bar{\omega})^{2}\right\}
$$

and the diffraction integral after the integration over the azimuthal angle reduces to [4]:

$$
\psi(E ; r)=\int_{0}^{\alpha} \exp \left(-\frac{i}{\hbar} g_{i} \chi_{a}(\vartheta, \delta)\right) J_{0}\left(\frac{g_{i} \vartheta r}{\hbar}\right) d \vartheta
$$

with $r=x^{2}+y^{2}$ being the distance from the axis in the image and $\vartheta=\sqrt{\omega \bar{\omega}}$. Moreover, if the Gaussian distribution in the virtual source is assumed, it is possible to reduce the convolution to the form of a simple 1-D integral

$$
j(r)=e^{-r^{2} / 2 \sigma^{2}} \int_{0}^{\infty} j_{P S F}(\tilde{r}) e^{\frac{-\tilde{r}^{2}}{2 \sigma^{2}}} J_{0}\left(\frac{i r \tilde{r}}{\sigma^{2}}\right) \tilde{r} \mathrm{~d} \tilde{r}
$$

The calculation procedure was implemented in Matlab and tested for a SLEEM system from the ISI. 


\section{Optimal aperture of the SLEEM}

For the calculation of the optimal aperture only axially symmetrical aberrations are relevant. The system we tested the algorithms on is a SLEEM developed at the ISI in cooperation with Delong Instruments. It consists of a magnetic condenser lens and an electrostatic objective lens; the presence of the magnetic field on the sample is undesirable (Figure 1). The electrons can be decelerated between the objective lens and the sample from the $5 \mathrm{keV}$ to units of volts. The Schottky emission source with the energy width of $0.8 \mathrm{eV}$ and the diameter of $20 \mathrm{~nm}$ is used. The optical properties of the system were calculated using the differential algebra (DA) method [5,6]. High order axial field derivatives needed by the DA was computed using the method described in [7].
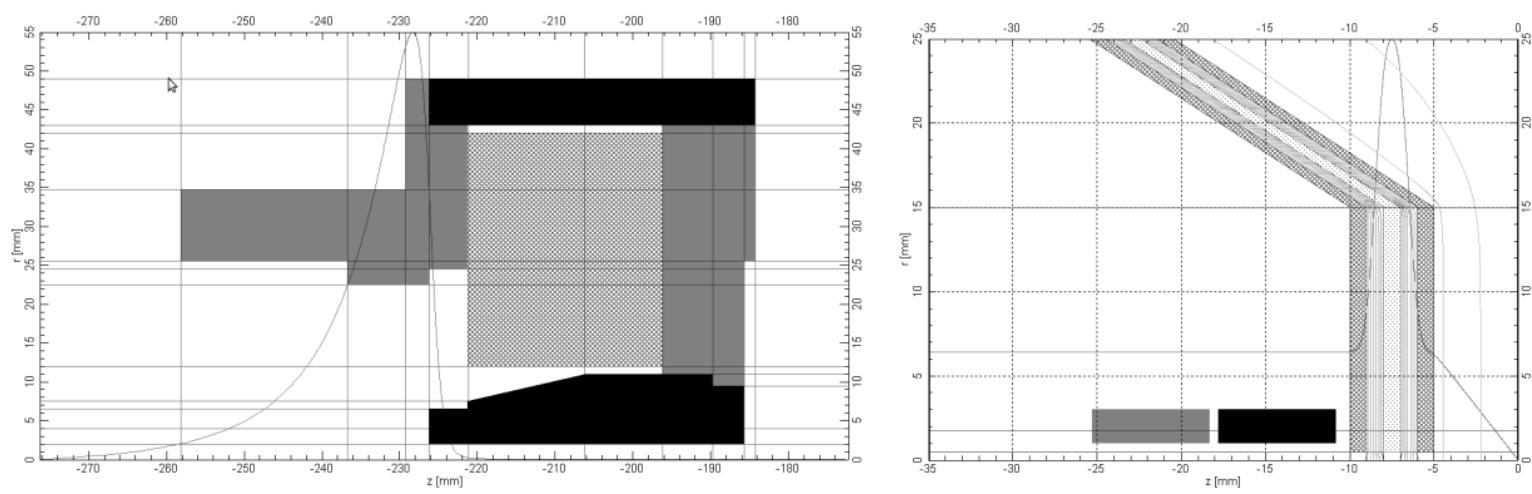

Figure 1. Design of the condenser magnetic lens and the objective electrostatic lens with the electrostatic double deflection system.

The typical longitudinal profiles are in Figure 2. The probe size is computed from the minimum of the curve $r_{50}$ defining the part of the beam containing $50 \%$ of the current. These profiles were calculated for several values of the aperture angle in the vicinity of the optimal aperture given by the Barth \& Kruit's approach to find the optimal aperture. The results are presented together with the optical properties of the system in the Table 1 and Figure 3. We can see that values of the optimal aperture angle computed from the wave optical agree with results of $\mathrm{B} \& \mathrm{~K}$ method. On the other hand the values of $d_{50}$ differ for small landing energies by about $40 \%$. It is caused mainly by the effect of the chromatic aberration of the second order which is not included in the $\mathrm{B} \& \mathrm{~K}$ approach and which is negative for low landing energies.
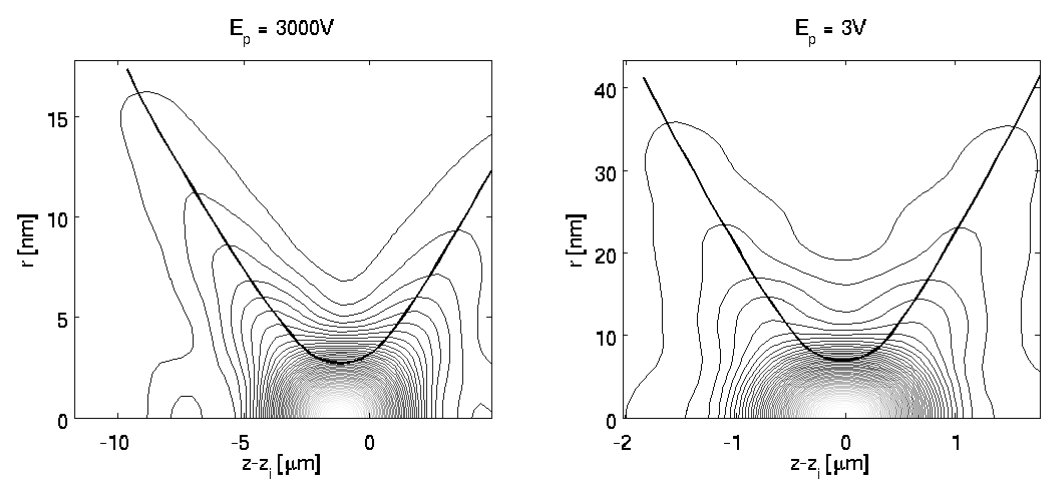

Figure 2. Examples of the longitudinal beam profile. The profile on the left was computed for the landing energy of $3 \mathrm{keV}$ and is influenced mainly by the spherical aberration, the other was computed for the landing energy of $3 \mathrm{eV}$ is influenced mainly by the chromatic aberrations 


\begin{tabular}{cccccccc}
\hline $\boldsymbol{E}[\boldsymbol{V}]$ & $\boldsymbol{d}_{\mathbf{5 0} \boldsymbol{w}}[\mathbf{n m}]$ & $\boldsymbol{\alpha}_{\boldsymbol{w}}[\boldsymbol{r a d}]$ & $\boldsymbol{d}_{\mathbf{5 0 \boldsymbol { B }} \boldsymbol{K}}[\mathbf{n m}]$ & $\boldsymbol{\alpha}_{\boldsymbol{B} \boldsymbol{K}}[\boldsymbol{r a d}]$ & $\boldsymbol{C}_{\mathbf{3}}[\boldsymbol{m}]$ & $\boldsymbol{C}_{\boldsymbol{C}}[\boldsymbol{m}]$ & $\boldsymbol{C}_{\boldsymbol{C 2}}[\boldsymbol{m}]$ \\
\hline 5000 & 4.80 & 0.0029 & 5.4428 & 0.0033 & $3.22 \mathrm{E}-01$ & $9.84 \mathrm{E}-03$ & $1.94 \mathrm{E}-02$ \\
3000 & 5.40 & 0.0034 & 6.1115 & 0.0038 & $2.35 \mathrm{E}-01$ & $5.81 \mathrm{E}-03$ & $6.35 \mathrm{E}-03$ \\
1000 & 6.43 & 0.0052 & 7.3893 & 0.0055 & $9.08 \mathrm{E}-02$ & $1.75 \mathrm{E}-03$ & $4.17 \mathrm{E}-04$ \\
500 & 7.10 & 0.0070 & 8.1088 & 0.0071 & $4.35 \mathrm{E}-02$ & $8.03 \mathrm{E}-04$ & $2.16 \mathrm{E}-05$ \\
100 & 8.33 & 0.0128 & 9.8154 & 0.0136 & $6.04 \mathrm{E}-03$ & $1.33 \mathrm{E}-04$ & $-2.06 \mathrm{E}-05$ \\
50 & 9.40 & 0.0167 & 10.7572 & 0.0176 & $2.40 \mathrm{E}-03$ & $6.22 \mathrm{E}-05$ & $-1.15 \mathrm{E}-05$ \\
25 & 10.13 & 0.0175 & 11.9341 & 0.0219 & $9.23 \mathrm{E}-04$ & $2.95 \mathrm{E}-05$ & $-6.03 \mathrm{E}-06$ \\
10 & 11.09 & 0.0247 & 13.8967 & 0.0281 & $2.54 \mathrm{E}-04$ & $1.12 \mathrm{E}-05$ & $-2.47 \mathrm{E}-06$ \\
5 & 12.05 & 0.0324 & 15.7424 & 0.0336 & $9.46 \mathrm{E}-05$ & $5.42 \mathrm{E}-06$ & $-1.24 \mathrm{E}-06$ \\
3 & 12.89 & 0.0386 & 17.3430 & 0.0382 & $4.55 \mathrm{E}-05$ & $3.20 \mathrm{E}-06$ & $-7.48 \mathrm{E}-07$ \\
2 & 13.58 & 0.0419 & 18.7810 & 0.0423 & $2.54 \mathrm{E}-05$ & $2.11 \mathrm{E}-06$ & $-4.99 \mathrm{E}-07$ \\
1 & 14.64 & 0.0508 & 21.6337 & 0.0503 & $9.42 \mathrm{E}-06$ & $1.04 \mathrm{E}-06$ & $-2.50 \mathrm{E}-07$ \\
\hline
\end{tabular}

Table 1. Optical properties of the SLEEM with respect to the landing energies
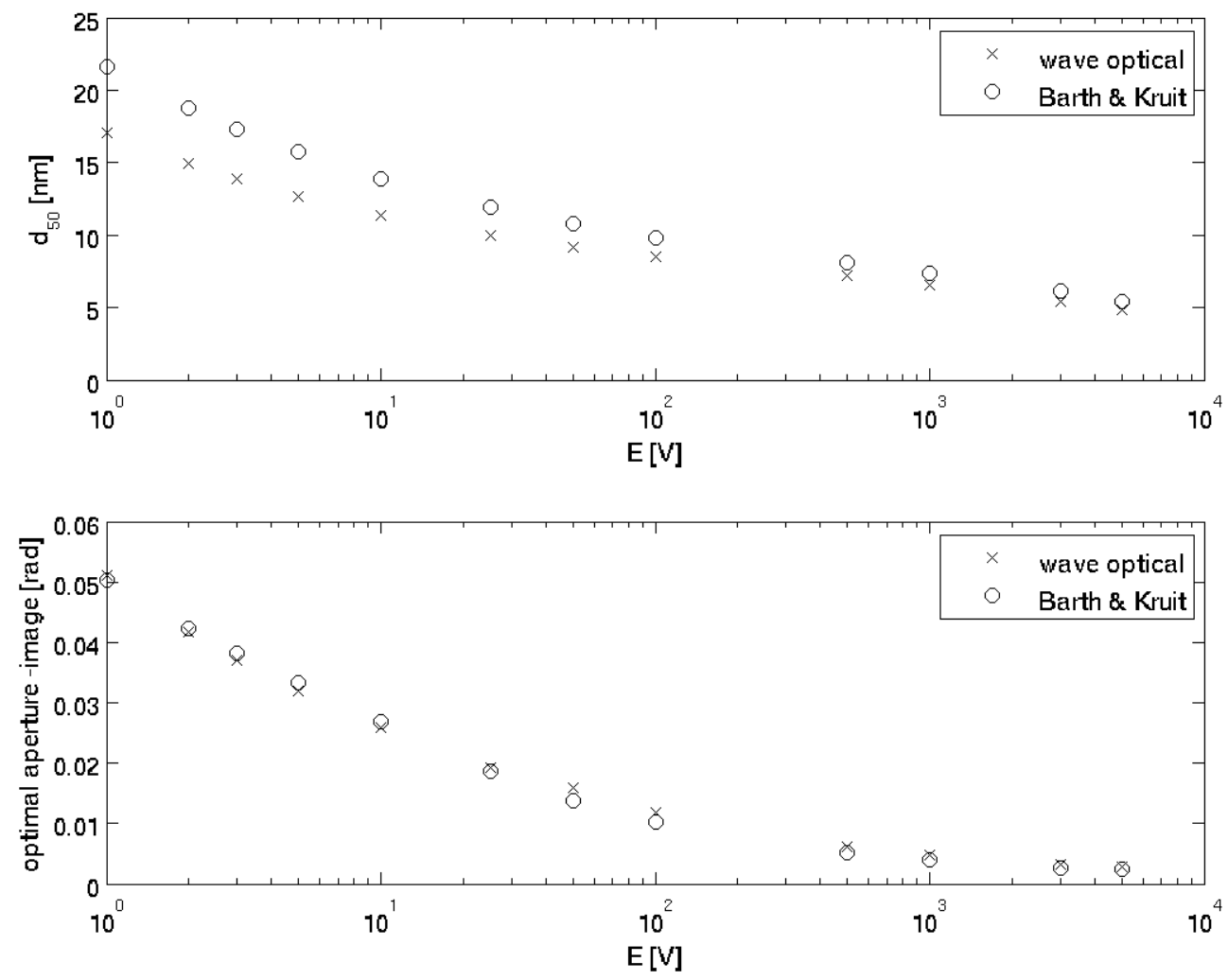

Figure 3. Comparison of the results from the wave optical calculations with those by the Bart \& Kruit formulas.

\section{Influence of the deflection aberrations}

The axial probe size determines the best possible resolution of the SEM, but it does not include the effect of the beam deflection during scanning the sample. The deflection is realized by the dipole fields 
that also influence the probe size. The effect depends on the field of view $(\mathrm{FoV})-$ the larger FoV the higher deflection is necessary, the stronger dipole field must be applied. The standard double deflection electrostatic system was assumed (Figure 1, right). The voltage of the deflector is set in such a way that the pivot point coincides with image nodal point of the objective.

A rigorous analysis of the deflection aberrations can be found in [8,9]. The aberration polynomial has an analogical form to the off-axial lens aberration polynomial, but the distance from the axis in the object is substituted by the deflection in the image. We will not derive the deflection aberration in this sense; we just calculate their effect for several FoVs. For each deflection the required deflection voltage is calculated, which is used for calculation of the axial aberrations. They are substituted to the general form of the aberration function and the PSF is calculated. Figure 4 shows typical current density profiles, the original symmetrical profile is blurred in the deflection direction. The effect increases with increasing FoV and decreasing landing energy as a result of the increasing dispersion effect, Figure 5.
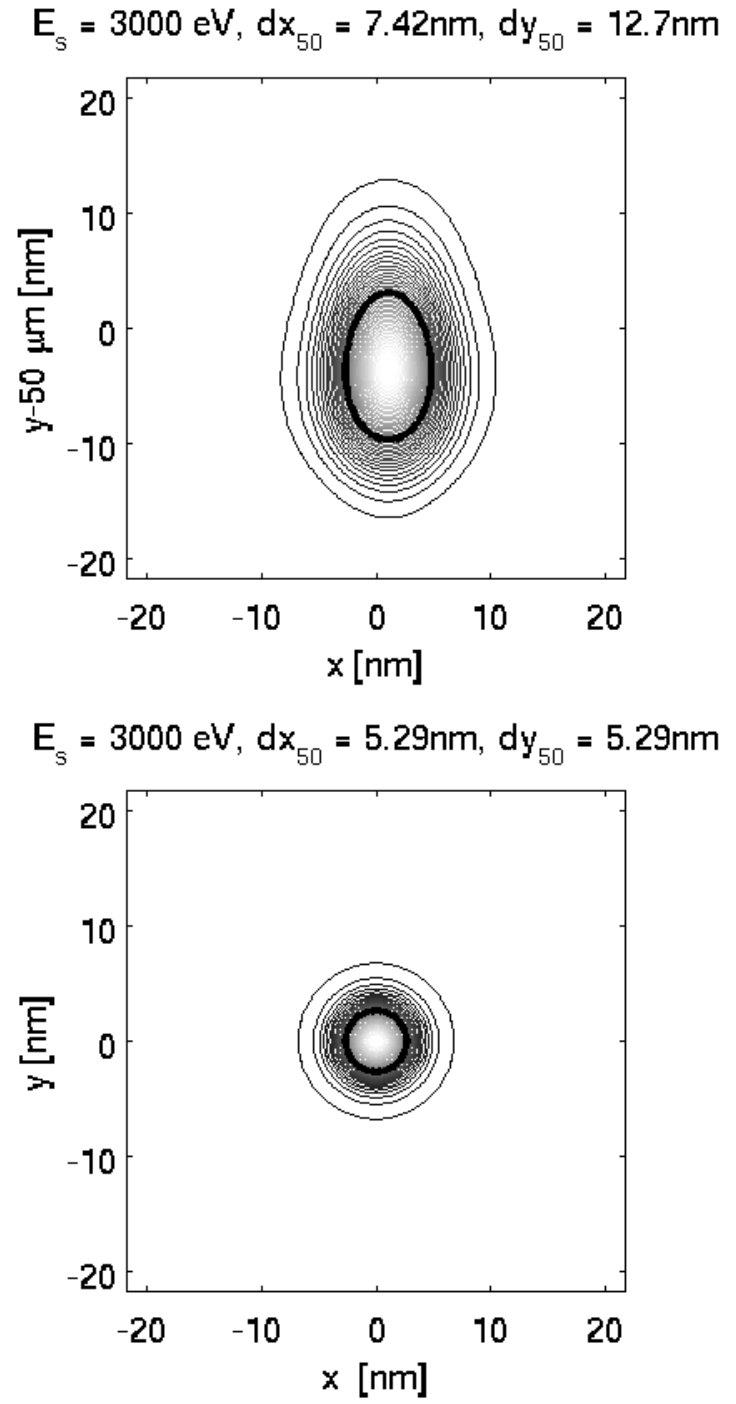

$E_{s}=3000 \mathrm{eV}, \mathrm{dx}_{50}=13.6 \mathrm{~nm}, \mathrm{dy}_{50}=13.6 \mathrm{~nm}$

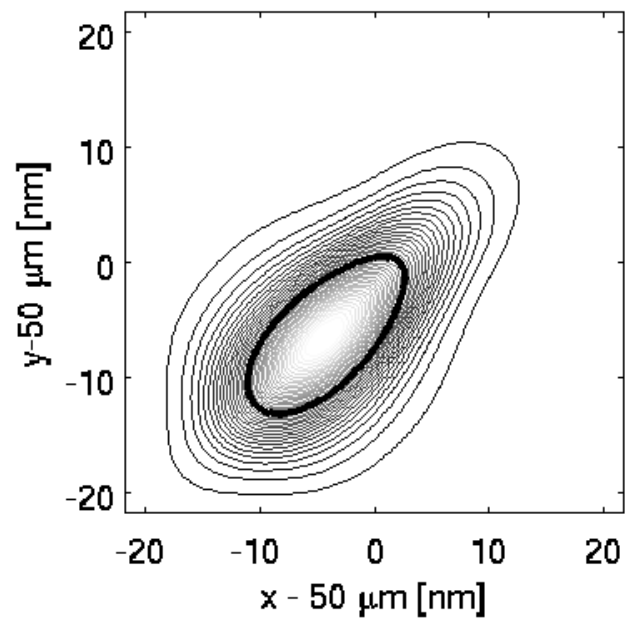

$E_{\mathrm{s}}=3000 \mathrm{eV}, \mathrm{dx}_{50}=12.7 \mathrm{~nm}, \mathrm{dy}_{50}=7.42 \mathrm{~nm}$

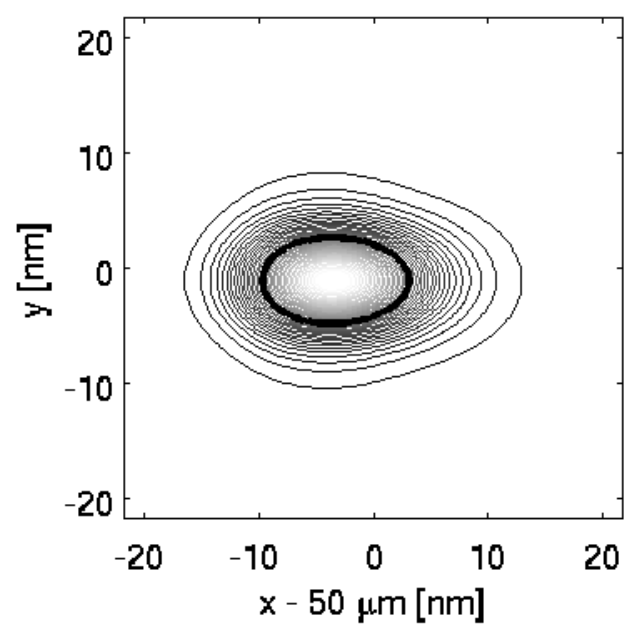

Figure 4. Current density beam profiles of the deflected beams in the plane of least confusion. The landing energy is $3 \mathrm{keV}$ and the FoV $100 \times 100 \mu \mathrm{m}^{2}$. 

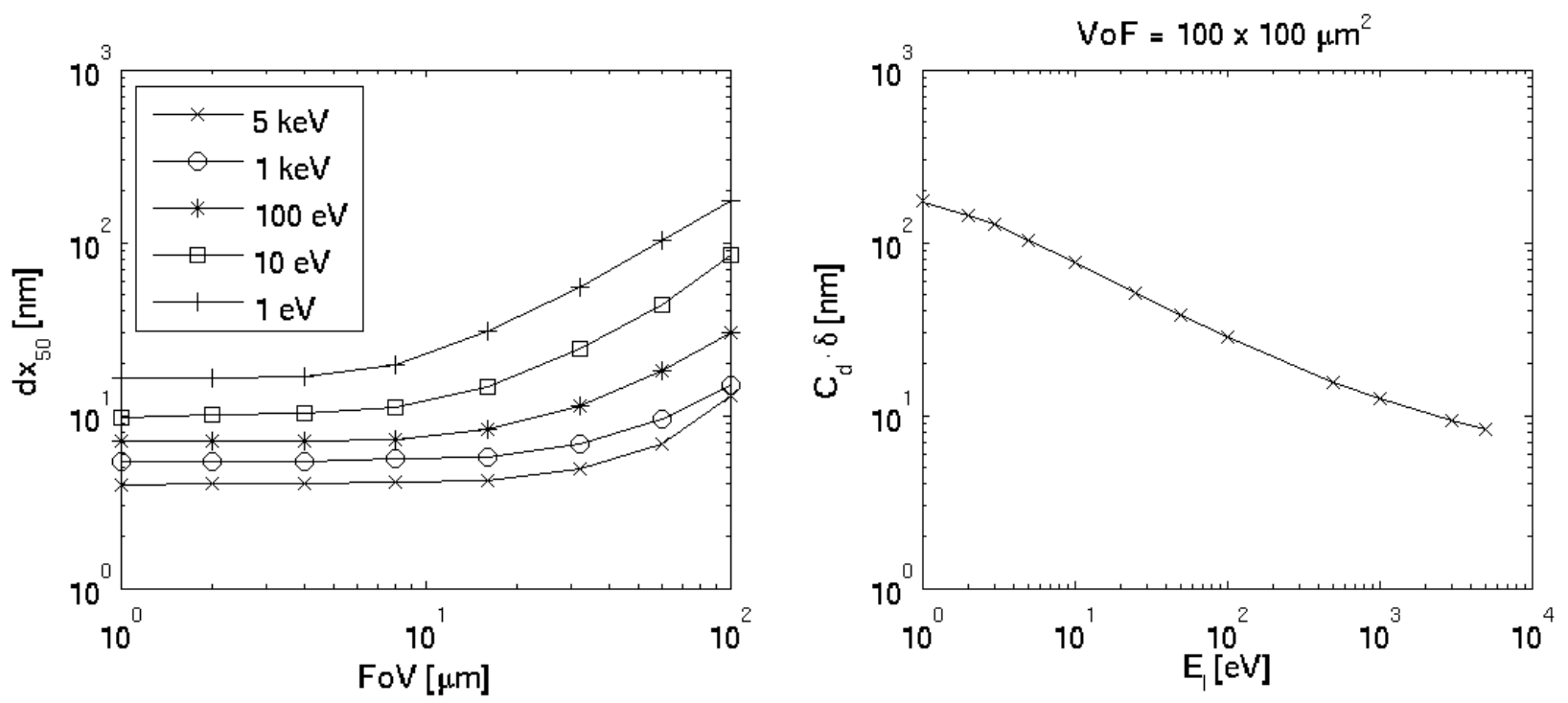

Figure 5. Influence of the deflection aberrations on the probe size for different energies and FoV (left) and the effect of the dispersion for the FoV $100 \times 100 \mu \mathrm{m}^{2}$ (right).

\section{Conclusion}

A wave-optical calculation of the probe size for the SLEEM was done. The comparison with the widelyused Barth and Kruit's approximation shows a very good agreement for high landing energies but there are higher differences in the low energy range. The disagreement can be, to the greater degree, explained by the influence of the second order chromatic aberration. The analysis of the deflection aberration was also carried out. It was shown that deflection aberrations reduce the number raster lines in a $100 \times$ $100 \mu \mathrm{m}^{2} \mathrm{FoV}$ to about 500 for the landing energy of $1 \mathrm{eV}$. [10]

\section{References:}

[1] J Barth and P Kruit, Optik 101 (1996), p. 101.

[2] PW Hawkes and E Kasper in "Principles of electron optics, Vol. 3", (Academic Press, London), 1994.

[3] M Haider, S Uleman and J Zach, Ultramicroscopy 81 (2000), p. 163.

[4] OL Krivanek et al in "Handbook of Charged Particle Optics", (CRC Press, Boca Raton), p. 601.

[5] M Berz, Adv. Imaging Electron Phys. 108 (1999).

[6] L Wang et al, Microelectron. Eng. 73-74 (2004), p. 90.

[7] T Radlička and B Lencová, Ultramicroscopy 110 (2010), p. 1198.

[8] E Goto and T Soma, Optik 48 (1977), p. 255.

[9] Y Kang et al, Optik 120 (2009), p. 591.

[10] The author acknowledges the support from Ministry of Education, Youth and Sports of the Czech Republic (LO1212 and 7H13015) and the European Commission (ALISI No. CZ.1.05/2.1.00/01.0017 and E450EDL, No. 325613). 